\title{
Erratum: Efficient electronic passivation scheme for computing low-symmetry compound semiconductor surfaces in density-functional theory slab calculations [Phys. Rev. Materials 5, 044605 (2021)]
}

\author{
Su-Hyun Yoo @ , Liverios Lymperakis $\odot$, and Jörg Neugebauer
}

(Received 4 November 2021; published 7 January 2022)

DOI: 10.1103/PhysRevMaterials.6.019901

After publication of our original paper on surface passivation on low-symmetry semiconductor surfaces, we became aware of the paper, Phys. Rev. Mater. 2, 073401 (2018), which proposed an alternative passivation approach (hereafter denoted as the zigzag approach). In this Erratum, we compare and contrast the two formalisms to clarify their differences in terms of targeted physical quantities and qualitative descriptions of surface electronic structures.

A key difference between the two passivation approaches are the targeted physical quantities: Whereas the authors of Ref. [1] are concerned about surface energies and thermodynamic stability, the aim of our passivation scheme is to get an efficient and accurate electronic structure description. The two very different targets, i.e., energetics vs electronic structure, result in qualitatively and conceptually different construction strategies and resulting structures.

The zigzag approach consists of the formation of nanofacets (zigzag structure) at the bottom side of the slab to reduce the density of surface sites suffering from what the authors of Ref. [1] call steric effects. To construct these structures and evaluate their energetics, the authors of Ref. [1] reported four steps: (1) Construct a conventional slab model with two conjugated semipolar surfaces, (2) modify the bottom surface of the slab into the corresponding zigzag structure, which should be then passivated by proper pseudo-H, (3) obtain the pseudochemical potentials (PCPs) of each pseudo-H used on the bottom surface, and especially for $a$-family ( $11 \overline{2} X)$ surfaces, estimate the PCPs of steric pseudo-H by well structures, and (4) estimate the absolute formation energy of the targeted surfaces by subtracting the total energy of the slab with the corresponding chemical potentials and PCPs of all the slab atoms.

The reconstruction inspired passivation scheme (RIPS), we proposed in our paper, consists of only two steps: In the first step, we follow the fundamental principles of surface reconstructions (i.e., electron counting rule, minimization of dangling bonds, etc.) to modify the stoichiometry at the bottom side. In the second step, we passivate the dangling bonds by pseudohydrogens with a valency that obeys the electron counting rule and compensates for the polarization-induced surface-bound charges. The two schemes to generate a passivated back side of a slab geometry are conceptually different and give rise to different geometries, as shown in the top panels [e.g., $(44 \overline{8} 5)$ and (112 2$)$ in Fig. 1]. In a few special cases, the atomic geometries of the passivated surfaces may become similar, typically when the length of the nanofacets becomes minimum and similar to the size of a typical surface reconstruction [e.g., (1012) in Fig. 1].

Since our approach does not require the formation of well-defined facets, it results, in general, in smoother geometries than the zigzag-shaped structures reported in Ref. [1]. More importantly, even for the (10 $\overline{1} 2)$ slabs where the geometries look similar, the two approaches provide a very different description of the electronic properties. RIPS compensates for the polarization-induced surface-bound charges and, thus, unambiguously describes the electronic structure of semipolar planes (e.g., see the surface band structure in the bottom and the right panel of Fig. 1). In contrast, the zigzag approach suffers from the spontaneous polarizationinduced field and introduces spurious states into the fundamental band gap (see the bottom and left panel of Fig. 1). These spurious states have severe consequences [2]: They give rise to a very poor slab size convergence and prevent the description of critical surface properties, such as surface bands or band gaps. 


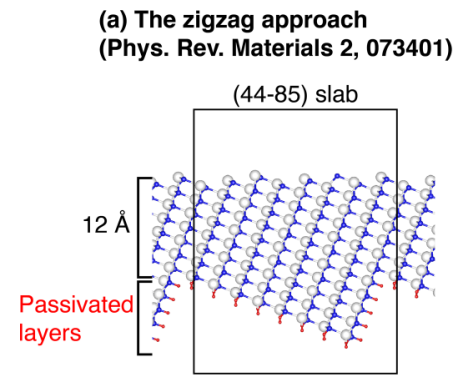

$(11-22)$ slab

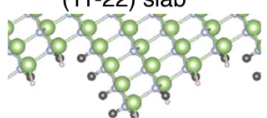

(10-12) slab

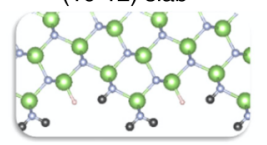

Surface band structure

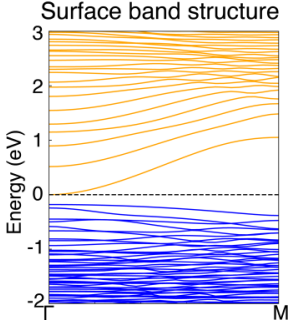

(b) The RIPS approach

(Phys. Rev. Materials 5, 044605)

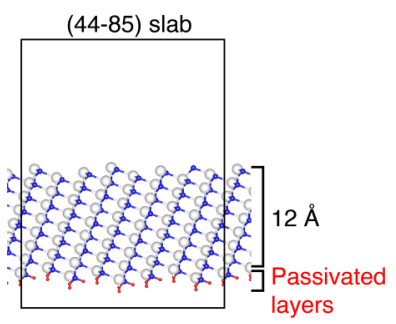

$(11-22)$ slab

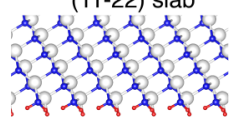

(10-12) slab
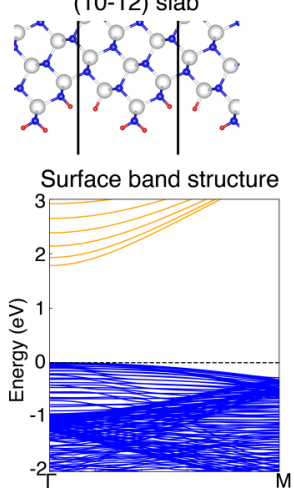

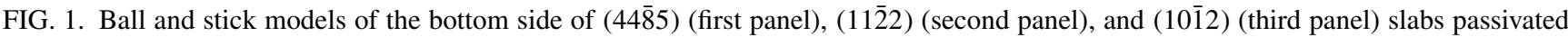
by the zigzag approach (left) and RIPS approach (right). The models in the second and third panels on the left are taken from Ref. [1]. Bottom (fourth) panel: Band structures of $\mathrm{GaN}(10 \overline{1} 2)$ slabs with a thickness of roughly $62 \AA$ where both surfaces are passivated by the zigzag approach (left) and by RIPS (right). Blue and yellow curves indicate valence and conduction bands, respectively. In both band structures, the highest occupied states are set to zero. The zigzag passivation is suffering from the polarization-induced field and introduces spurious states into the fundamental band gap. RIPS compensates for the polarization-induced surface-bound charges and unambiguously describes the electronic properties of the semipolar surfaces.

[1] Y. Zhang, J. Zhang, and J. Zhu, Phys. Rev. Mater. 2, 073401 (2018).

[2] S.-H. Yoo, M. Todorova, D. Wickramaratne, L. Weston, C. G. Van de Walle, and J. Neugebauer, npj Comput. Mater. 7, 58 (2021). 